\title{
Stark Resonances: Asymptotics and Distributional Borel Sum ${ }^{\star}$
}

\author{
E. Caliceti ${ }^{1}$, V. Grecchi ${ }^{1}$, and M. Maioli ${ }^{2}$ \\ ${ }^{1}$ Dipartimento di Matematica, Università di Bologna, I-40127 Bologna, Italy \\ 2 Dip. di Matematica Pura e Applicata, Università di Modena, I-41100 Modena, Italy
}

Received November 4, 1992, in revised form December 15, 1992

\begin{abstract}
We prove that the Stark effect perturbation theory of a class of bound states uniquely determines the position and the width of the resonances by Distributional Borel Sum. In particular the small field asymptotics of the width is uniquely related to the large order asymptotics of the perturbation coefficients. Similar results apply to all the "resonances" of the anharmonic and double well oscillators.
\end{abstract}

\section{Introduction}

Distributional Borel (DB) summability was defined in [7], following a suggestion of 't Hooft [23] for double well problems. In particular a criterion for summability was proved [7] and some applications were performed to lattice field theories and to double well problems $([7,8])$ and to the justification of the semiclassical method [9].

Here we prove the DB summability of the perturbation series for the resonances of anharmonic oscillators of unstable anharmonic oscillators and of the Hydrogen Stark effect resonances. More precisely we should say that each resonance is directly given by the lower DB sum, or that the position is given by the DB sum itself and the width by the modulus of the DB discontinuity. With respect to the previous result [14] of complex field Borel summability (and continuation to the real axis), this yields a more direct connection between perturbation series and resonances. In fact now we are able to connect uniquely all the types of asymptotics introduced by Bender and $\mathrm{Wu}[2,3,4]$ for the anharmonic oscillator case (also see $[16,5]$; for other references see [21, 22]). In such a way we extend the well known Herbst-Simon connection formulas. Such connection is clarified by the first singularity on the positive half-axis of the Borel transform of the perturbation series. The kind of this singularity agrees with the one suggested by Ecalle's theory of resurgent functions [12].

* Partially supported by Ministero della Università e della Ricerca Scientifica 
The proof proceeds the following way. First we prove that the anharmonic oscillator "resonances" are analytic in some Nevanlinna disk and then we show the same result for a class of Stark effect resonances. As for the method of the proof, Hunziker's theory of Schrödinger eigenvalue stability $([18,19])$ and subsequent extensions by Caliceti and Maioli $[11]$ and all of us $([8,10])$ are combined with a suitable version of an "energy bound."

DB summability follows from the known ordinary Borel summability in contiguous sectors plus the analyticity extension result. Equivalence of the different types of asymptotics is proved by application of the direct and inverse Borel transform.

Finally, all these results apply to the double well "resonances," too, which are in fact related to quartic anharmonic oscillator "resonances" for particular values of the parameters $([13,1,6,8,10])$.

\section{Analyticity of the Anharmonic Oscillator Eigenvalues in the Nevanlinna Disk}

Consider the quartic $d$-dimensional anharmonic oscillator in $L^{2}\left(\mathbf{R}^{d}\right)$ with coupling constant $g^{2}=\rho^{2} \exp (2 i \theta)$ :

$$
-\Delta+\mathbf{x}^{2}+g^{2} \mathbf{x}^{4}
$$

For each fixed eigenvalue $E_{n}(g)$ and for any $\varepsilon_{0}>0$, there is $\rho_{0}>0$ such that $E_{n}(g)$ is analytic (see [20] and related references) in the sector

$$
\left\{g:-3 \pi / 2+2 \varepsilon_{0} \leqq 2 \theta \leqq 3 \pi / 2+2 \varepsilon_{0},|g| \leqq \rho_{0}\right\} .
$$

We look for the behaviour, as $\rho \rightarrow 0$, of a minimal infinitesimal function $\varepsilon=\varepsilon(\rho)$, such that the eigenvalues are analytic for

$$
-3 \pi / 4+\varepsilon(\rho) \leqq \theta \leqq 3 \pi / 4-\varepsilon(\rho), \quad 0<\rho \leqq B
$$

for some $B$ independent of $\theta$. Setting $\theta=-3 \pi / 4+\varepsilon$ and scaling $x \rightarrow \lambda x$, with $\lambda=e^{-i \theta / 3}$, it is sufficient to study the radial part of such an operator in the case of angular momentum $l$, i.e.

$$
A(\rho)=e^{i(-\pi / 2+2 \varepsilon / 3)}\left\{p^{2}+\frac{\left(j^{2}-1\right)}{4 r^{2}}\right\}+e^{i(\pi / 2-2 \varepsilon / 3)} r^{2}+\rho^{2} e^{i(-\pi / 2+2 \varepsilon / 3)} r^{4}
$$

in $L^{2}\left(\mathbf{R}^{+}\right), p=-i \frac{d}{d r}, j=d+2(l-1), j \geqq 0$ or $j=-1$, with suitable conditions at the origin. We are going to prove that the eigenvalues are in fact analytic in the region (3) if

$$
\varepsilon(\rho):=\frac{1}{2} R^{-1} \rho^{2}
$$

is chosen with $R$ sufficiently small. Such a property is easily checked to be equivalent to analyticity in the disk of radius $R / 2$ tangent in the origin to the imaginary axis of the $g^{2}$-plane, as stated in the following

Theorem 1. For each eigenvalue $E_{n}, n=0,1,2, \ldots$, of the quartic oscillator (1), there is $R>0$ such that $E_{n}(g)$ is analytic in the Nevanlinna disk $\Re g^{-2} \leqq-R^{-1}$ of the $g^{2}$-plane. 
Of course the theorem is true if level crossings of the eigenvalues do not exist inside such a disk for $R$ sufficiently small; since any given disk is the union of the boundaries of disks of smaller radius, the proof reduces to a stability argument for the operator family (4) as $g$ tends to zero along the boundaries asymptotically specified in (4b).

First let us consider a transformation $U$ in the set of $L^{2}$ functions which are translation analytic in some suitable strip $|\Im r|<\delta$ (for fixed $\eta_{0}, 0<\eta_{0}<1$ ):

$$
U f(r)=\xi^{\prime}(r)^{1 / 2} f(\xi(r)),
$$

where, setting $r_{0}=(\rho \sqrt{2})^{-1}$ for any fixed $\rho>0$, we define $\xi(r)$ in $C^{\infty}\left(R^{+}\right)$so that

$$
\begin{gathered}
\xi(r)=r-2 i \eta_{0}+2 i \eta_{0}\left(1+r^{3}\right)^{-1 / 6}, \quad 0<r \leqq r_{0} ; \\
\xi(r)=r, \quad r \geqq r_{0}+\eta_{0} .
\end{gathered}
$$

Setting $f(r)=\left(\xi^{\prime}(r)\right)^{-1}$, the transformed operator $U A(\rho) U^{-1}$ is given by

$$
H_{\rho}=\alpha\left[p f(r)^{2} p+4^{-1}\left(f(r)^{2}\right)^{\prime \prime}\right]+\alpha \frac{j^{2}-1}{4 \xi(r)^{2}}+\alpha^{-1} \xi(r)^{2}+\alpha \rho^{2} \xi(r)^{4},
$$

where $\alpha=e^{i(-\pi / 2+2 \varepsilon / 3)}$. The quadratic form which generates the operator $H_{\rho}$ is defined by:

$$
h_{\rho}[u]=\int_{0}^{\infty}\left\{\alpha f^{2}|p u|^{2}+\left[\alpha \frac{j^{2}-1}{4 \xi^{2}}+\alpha 4^{-1}\left(f^{2}\right)^{\prime \prime}+\alpha^{-1} \xi^{2}+\alpha \rho^{2} \xi^{4}\right]|u|^{2}\right\} d r
$$

on the maximal domain with the condition at the origin: $u(r) \simeq r^{(1+j) / 2}$.

We notice that the limit in the strong resolvent sense, as $\rho \rightarrow 0^{+}$, of $H_{\rho}$ is well defined by

$$
H_{0}=-i\left\{p f_{0}^{2} p+4^{-1}\left(f_{0}^{2}\right)^{\prime \prime}\right\}-\frac{i\left(j^{2}-1\right)}{4 \xi_{0}^{2}}+i \xi_{0}^{2},
$$

where $f_{0}(r)=\left(\xi_{0}^{\prime}(r)\right)^{-1}$ and the expression in (5b) provides $\xi_{0}(r), \forall r>0$. From now on, let $k=4^{-1}\left(j^{2}-1\right)$.

The proof of Theorem 1 consists in obtaining a stability result for the eigenvalues of $H_{0}$ with respect to the family $\left\{H_{\rho}\right\}_{\rho}>0$. This is achieved on the basis of a preliminary estimate (a kind of position-dependent energy bound).

Lemma 2. Let $\varepsilon=\frac{1}{2} R^{-1} \rho^{2}$. There is $\rho_{0}>0$ and there are positive constants $a_{1}, a_{2}, c, c_{1}, c_{2}$ such that

$$
\begin{aligned}
\Re h_{\rho}[u] \geqq & a_{1} \int_{1}^{r_{0}}\left\{\varepsilon\left[1-r^{4}\left(1+r^{3}\right)^{-7 / 3}\right]+\eta_{0} r^{2}\left(1+r^{3}\right)^{-7 / 6}\right\}|p u|^{2} d r \\
& +a_{2} \int_{r_{0}}^{\infty} \varepsilon|p u|^{2} d r-c\|u\|^{2}
\end{aligned}
$$

uniformly for $0<\rho \leqq \rho_{0}, u \in D\left(h_{\rho}\right)$. A similar estimate holds with $h_{\rho}$ replaced by $h_{0}\left(r_{0}=+\infty, \varepsilon=0\right)$.

Moreover, $\forall u$ :supp $u \subset(n,+\infty)$,

$$
\mathfrak{R} h_{\rho}[u] \geqq\left(c_{1} R^{-1}-c_{2}\right)\|u\|^{2}
$$

for $n$ large and $c_{1}, c_{2}$ independent of $R, 0 \leqq \rho \leqq \rho_{0}$. 
Proof. We have

$$
\begin{aligned}
\int_{0}^{\infty}\left\{f^{2}|p u|^{2}+k \xi^{-2}|u|^{2}\right\} d r= & \int_{0}^{\infty} f^{2}\left[|p u|^{2}+k r^{-2}|u|^{2}\right] d r \\
& +\int_{0}^{\infty} k\left[\xi^{-2}-f^{2} r^{-2}\right]|u|^{2} d r .
\end{aligned}
$$

Since the second integral on the right-hand side of (11) is bounded by const. $\|u\|^{2}$,

$$
\begin{aligned}
\mathfrak{R} \int_{0}^{\infty}\left\{f^{2}|p u|^{2}+k \xi^{-2}|u|^{2}\right\} d r & \geqq \int_{0}^{\infty} \mathfrak{R}\left(\alpha f^{2}\right)\left[|p u|^{2}+k r^{-2}|u|^{2}\right] d r-\text { const. }\|u\| \\
& =\int_{0}^{1}(\ldots)+\int_{1}^{r_{0}}(\ldots)+\int_{r_{0}}^{\infty}(\ldots)-\text { const. }\|u\|^{2}
\end{aligned}
$$

with the obvious meaning of the notation.

Next, for $r \leqq r_{0}$, notice that

$$
\begin{aligned}
\mathfrak{R} f^{2} & =\left\{1-\eta_{0}^{2} r^{4}\left(1+r^{3}\right)^{-7 / 3}\right\}\left|1-i \eta_{0} r^{2}\left(1+r^{3}\right)^{-7 / 6}\right|^{-4} \\
& \geqq 4^{-1}\left\{1-r^{4}\left(1+r^{3}\right)^{-7 / 3}\right\} \\
\mathfrak{F} f^{2} & =2 \eta_{0} r^{2}\left(1+r^{3}\right)^{-7 / 6}\left|1-i \eta_{0} r^{2}\left(1+r^{3}\right)^{-7 / 6}\right|^{-4} \\
& \geqq 2^{-1} \eta_{0} r^{2}\left(1+r^{3}\right)^{-7 / 6}
\end{aligned}
$$

Hence

$$
\begin{aligned}
\mathfrak{R}\left(\alpha f^{2}\right) & =(\mathfrak{R} \alpha)\left(\mathfrak{R} f^{2}\right)-(\mathfrak{F} \alpha)\left(\mathfrak{F} f^{2}\right) \\
& \geqq 4^{-1} \sin \left(\frac{2 \varepsilon}{3}\right)\left[1-r^{4}\left(1+r^{3}\right)^{-7 / 3}\right]+\frac{\eta_{0}}{2} \cos \left(\frac{2 \varepsilon}{3}\right) r^{2}\left(1+r^{3}\right)^{-7 / 6}
\end{aligned}
$$

Now, since the integral from 0 to 1 in (12) is nonnegative, using (15) for $\int_{1}^{r_{0}}$ and (5c) for $\int_{r_{0}}^{\infty}$, we get:

$$
\begin{aligned}
\mathfrak{R}\left\{\alpha \int_{0}^{\infty}\left[f^{2}|p u|^{2}+k \xi^{-2}|u|^{2}\right] d r\right\} \geqq & k_{1} \int_{r_{0}}^{\infty} \varepsilon|p u|^{2} d r-k_{2}\|u\|^{2} \\
& +k_{3} \int_{1}^{r_{0}}\left\{\varepsilon\left[1-r^{4}\left(1+r^{3}\right)^{-7 / 3}\right]\right. \\
& \left.+\eta_{0} r^{2}\left(1+r^{3}\right)^{-7 / 6}\right\}|p u|^{2} d r
\end{aligned}
$$

for some $k_{1}, k_{2}, k_{3}>0, \forall u \in D\left(h_{\rho}\right)$.

As for the remaining terms appearing in $\mathfrak{R} h_{\rho}[u],(7)$, notice that $\left(f^{2}\right)^{\prime \prime}$ can be neglected since it is bounded. Moreover, fixing $R>0$, for any $b>0$ there is $c>0$ such that

$$
r \in(0, b) \Rightarrow \mathfrak{R}\left\{\alpha^{-1} \xi(r)^{2}+\alpha \rho^{2} \xi(r)^{4}\right\} \geqq-c
$$


Choosing $b>0$ such that, within $\left(b, r_{0}\right), \mathfrak{R}\left\{\alpha^{-1} \xi(r)^{2}+\alpha \rho^{2} \xi(r)^{4}\right\}$ attains its minimum in $r_{0}$, we have

$$
\begin{aligned}
r \in\left(b, r_{0}+\eta_{0}\right) \Rightarrow & \Re\left\{\alpha^{-1} \xi(r)^{2}+\alpha \rho^{2} \xi(r)^{4}\right\} \\
\geqq & r^{2} \sin (2 \varepsilon / 3)+\rho^{2} r^{4} \sin (2 \varepsilon / 3)+4 r \eta_{0} \cos (2 \varepsilon / 3) \\
& -8 r^{3} \eta_{0} \rho^{2} \cos (2 \varepsilon / 3) \geqq c_{1} R^{-1},
\end{aligned}
$$

where $c_{1}$ is independent of $R$ (and, of course, of $b$ ). Notice that (18) is obtained using the definition of $\xi(r)$ and the assumption $\varepsilon=(2 R)^{-1} \rho^{2}$. As a consequence of (18),

$$
r \in\left(r_{0}+\eta_{0},+\infty\right) \Rightarrow \mathfrak{R}\left\{\alpha^{-1} \xi(r)^{2}+\alpha \rho^{2} \xi(r)^{4}\right\} \geqq c_{1} R^{-1}
$$

since such a real part turns out to be increasing in that half line. Of course (17), (18), (19) hold uniformly for $0<\rho \leqq \rho_{0}$ for some $\rho_{0}=\rho_{0}(R)>0$ and the lemma is proved.

Corollary 3. Let $\chi_{n}(r)=\chi(r / n), \chi \in C^{\infty}\left(\mathbb{R}^{+}\right)$and $\chi(r)=1$ for $r \leqq 1, \chi(r)=0$ if $r \geqq 3 / 2$. Then $\exists c_{3}>0$ such that

$$
\left\|\left[H_{\rho}, \chi_{n}\right] u\right\| \leqq c_{3} n^{-1 / 4}\left(\left\|H_{\rho} u\right\|+\|u\|\right)
$$

$\forall u \in D\left(H_{\rho}\right), 0 \leqq \rho \leqq \rho_{0}$.

Proof. It is enough to prove (20) for $\rho>0$, since for $\rho=0$ the argument is even simpler. For simplicity, let $u \in D\left(H_{\rho}\right)$ be such that $\|u\|=1$. Let $\gamma_{2 n}$ be the characteristic function of $[1,2 n]$. By the choice of $\chi$,

$$
\left.\left[H_{\rho}, \chi_{n}\right]=-\gamma_{2 n}\left\{\alpha f^{2} 2 i n^{-1} \chi^{\prime}\left(n^{-1} r\right) p+n^{-2} \chi^{\prime \prime}\left(n^{-1} r\right)\right]+2 \alpha n^{-1} f^{\prime} f \chi^{\prime}\left(n^{-1} r\right)\right\} \text {. }
$$

Thus

$$
\begin{aligned}
\left\|\left[H_{\rho}, \chi_{n}\right] u\right\| & \leqq c_{4} n^{-1}\left\{\int_{1}^{2 n}|p u|^{2} d r\right\}^{1 / 2} \\
& \leqq c_{5}\left(1+8 n^{3}\right)^{7 / 12}(2 n)^{-1} \dot{n}^{-1}\left\{\int_{1}^{2 n}|p u|^{2} \eta_{0} r^{2}\left(1+r^{3}\right)^{-7 / 6} d r\right\}^{1 / 2} \\
& \leqq c_{6} n^{-1 / 4}\left\{\int_{1}^{r_{0}} \eta_{0} r^{2}\left(1+r^{3}\right)^{-7 / 6}|p u|^{2} d r\right\}^{1 / 2} \\
& \leqq c_{7} n^{-1 / 4}\left\{\Re\left\langle H_{\rho} u, u\right\rangle+1\right\},
\end{aligned}
$$

where the last inequality follows from Lemma 2. This concludes the proof.

Remark. By a direct analysis of the adjoint operator $H_{\rho}^{*}$ it is not difficult to obtain the analogous estimate

$$
\left\|\left[H_{\rho}^{*}, \chi_{n}\right] u\right\| \leqq c_{3} n^{-1 / 4}\left(\left\|H_{\rho}^{*} u\right\|+\|u\|\right)
$$

$\forall u \in D\left(H_{\rho}^{*}\right), 0 \leqq \rho \leqq \rho_{0}$.

\section{Proposition 4. Let}

$$
M_{n}=1-\chi_{n}
$$

where $\chi_{n}$ is defined in Corollary 3. If

$$
d_{n}(\lambda, \rho):=\inf \left\{\left\|\left(\lambda-H_{\rho}\right) M_{n} u\right\|:\left\|M_{n} u\right\|=1, u \in D(H(\rho))\right\},
$$


then $\forall \lambda \in \mathbf{C}, \exists R>0, \exists n_{0}, \rho_{0}, \delta>0$ such that in the Nevanlinna disk $\mathfrak{R}^{-2}$ $<-R^{-1}$

$$
d_{n}(\lambda, \rho) \geqq \delta>0, \quad \forall n \geqq n_{0}, \quad \forall \rho \leqq \rho_{0}
$$

Proof. By (10)

$$
\Re\left\langle M_{n} u, H_{\rho} M_{n} u\right\rangle \geqq\left\langle M_{n} u, c_{1} R^{-1} M_{n} u\right\rangle-c_{2} \text {. }
$$

Now $c_{1} R^{-1}$ can be made large enough so that (25) holds, by choosing $R$ sufficiently small (of course $\mathrm{R}$ will tend to infinity like $|\lambda|^{-1}$ as $\lambda \rightarrow 0$ ).

Remark. The estimate (26) is an indication for the following conjecture: the radius of the two disks, tangent to each other, between which the Bender-Wu horn of singularities occurs, tends to infinity like $E_{0}(0)^{-1}$, where $E_{0}(g)$ is the ground state energy. This clearly appears as $j \rightarrow-2$ in (4a), where $E_{0}(g) \rightarrow 0, \forall g$.

Lemma 5. Let the two sequences $\rho_{m}>0, u_{m} \in D\left(H_{\rho_{m}}\right)$ be given so that $\rho_{m} \rightarrow 0$, $\left\|H_{\rho_{m}} u_{m}\right\|$ is bounded and $\left\|u_{m}\right\|=1, u_{m}$ tends weakly to 0 . Then the sequences $\rho_{m(n)}, M_{n} u_{m(n)}$ satisfy the same properties for some suitable $m=m(n)$ if $R$ is chosen sufficiently small.

Proof. The boundedness of $\left\|H_{\rho_{m}} M_{n} u_{m}\right\|$ reduces to the boundedness of $\left\|H_{\rho_{m}} u_{m}\right\|$ by virtue of Corollary 3 . Thus it is enough to prove that for all $n$

$$
\lim _{n}\left\|\chi_{n} u_{m(n)}\right\|=0 \text {. }
$$

To prove (31), let $H_{\rho}^{\prime}=\alpha^{-1} H_{\rho}$ and $\lambda \in \mathbf{C}-\sigma\left(H_{0}^{\prime}\right)$ be fixed. Then

$$
\left\|\chi_{n} u_{m}\right\|^{2} \leqq c\left(\left\|\chi_{n} R_{0}^{\prime}\left(H_{0}^{\prime}-H_{\rho_{m}}^{\prime}\right) u_{m}\right\|^{2}+\left\|\chi_{n} R_{0}^{\prime}\left(H_{\rho_{m}}^{\prime}-\lambda\right) u_{m}\right\|^{2}\right) .
$$

The second term tends to zero because $R_{0}^{\prime} \equiv\left(H_{0}^{\prime}-\lambda\right)^{-1}$ is compact and $\left(H_{\rho_{m}}^{\prime}-\lambda\right) u_{m}$ tends weakly to zero. Calling $\gamma_{2 n}$ the characteristic function of $[0,2 n]$, the first term is bounded, up to some constant factor, by

$$
\left\|\gamma_{2 n} R_{0}^{\prime} \chi_{n}\left(H_{0}^{\prime}-H_{\rho_{m}}^{\prime}\right) u_{m}\right\|^{2}+\left\|\gamma_{2 n}\left[R_{0}^{\prime}, \chi_{n}\right]\left(H_{0}^{\prime}-H_{\rho_{m}}^{\prime}\right) u_{m}\right\|^{2} \text {. }
$$

In turn the first term in (29) can be bounded as follows:

$$
\begin{gathered}
c \int_{0}^{2 n}\left|R_{0}^{\prime} \chi_{n}\left(H_{0}^{\prime}-H_{\rho_{m}}^{\prime}\right) u_{m}\right|^{2} d r \leqq c\left\|R_{0}^{\prime}\right\|^{2} \int_{0}^{2 n}\left|\left(H_{0}^{\prime}-H_{\rho_{m}}^{\prime}\right) u_{m}\right|^{2} d r \\
=c\left\|R_{0}^{\prime}\right\|^{2} \int_{0}^{2 n}\left(|\xi(r)|^{2}\left|1-e^{-4 i \varepsilon_{m} / 3}\right|+\rho_{m}^{2}|\xi(r)|^{4}\right)^{2} d r \\
\leqq c^{1}\left(\varepsilon_{m} n^{2}+\rho_{m}^{2} n^{4}\right)^{2}\left\|u_{m}\right\|^{2}
\end{gathered}
$$

where $\varepsilon_{m}=\frac{1}{2} R^{-1} \rho_{m}^{2}$ by hypothesis. As for the second summand of (29) we have:

$$
\left\|\gamma_{2 n} R_{0}^{\prime} \chi_{n}\left(H_{0}^{\prime}-H_{\rho_{m}}^{\prime}\right) u_{m}\right\| \leqq\left\|R_{0}^{\prime}\left[H_{0}^{\prime}, \chi_{n}\right] R_{0}^{\prime}\left(H_{0}^{\prime}-H_{\rho_{m}}^{\prime}\right) u_{m}\right\| \leqq \tilde{c} n^{-1 / 4} .
$$

Indeed by (23) the operator $R_{0}^{\prime}\left[H_{0}^{\prime}, \chi_{n}\right]=\left(\left[\chi_{n},\left(H_{0}^{\prime}\right)^{*}\right]\left(R_{0}^{\prime}\right)^{*}\right)^{*}$, when applied to the bounded sequence $R_{0}^{\prime}\left(H_{0}^{\prime}-H_{\rho_{m}}^{\prime}\right) u_{m}$, satisfies the last inequality in (31).

Proof of Theorem 1. The theorem follows from the preceding propositions by applying the eigenvalue stability theory as in $[18,19,11,10,8]$. In particular the essential hypotheses are satisfied by virtue of Corollary 3 and Lemma 5. 


\section{Analyticity of Stark Effect Resonances in a Nevanlinna Disk}

As is well known $([14,17])$, the Stark effect operator

$$
-\Delta-|x|^{-1}+F x_{1}
$$

has proper eigenvalues for $\mathfrak{F} F>0$, which admit an analytic continuation, for small $|F|$, to any phase preceding $\arg (F)=3 \pi / 2$ (or $\arg (F)=-\pi / 2$ ).

To prove DB summability in the real direction we want to extend the analyticity region and the asymptotics of the Stark resonances.

Theorem 6. The Stark resonances $E_{k, k, m}(F), k=0,1,2, \ldots, m=0, \pm 1, \pm 2, \ldots$ admit an analytic continuation from $\mathfrak{F} F>0,|F|<z_{0}$ to a Nevanlinna disk:

$$
D_{z_{0}}=\left\{z:\left|z-z_{0}\right|<z_{0}\right\}, \quad\left(\text { if } z \in D_{z_{0}},-z \notin D_{z_{0}}\right)
$$

for some radius $z_{0}>0$.

Proof. Consider the ordinary operators associated with (32) and let $\mu(\beta)=\mu_{k}^{m}(\beta)$ be the $k^{\text {th }}$ eigenvalue of $-\frac{d^{2}}{d x^{2}}+\left(m^{2}-1 / 4\right) \frac{1}{x^{2}}+x^{2}+\beta x^{4}, x \in R^{+}$. We define $f(\beta)=\mu(\beta)+\mu\left(e^{-i \pi} \beta\right)$ and we have $[14]$ :

$$
\eta=f(\beta), \text { where } \eta=(-2 E)^{-1 / 2}, \quad \beta=F \eta^{3} .
$$

By the results of the preceding section $f(\beta)$ is analytic in $D_{z_{0}}$ with image $\Omega$ contained in $\Omega_{1}$ defined by:

$$
\Omega_{1}=\left\{z:\left|z-\eta_{0}-\eta_{1} \beta^{2}\right| \leqq C \beta^{4}, \quad \forall \beta \in D_{z_{0}}, \eta_{0}>0, \eta_{1} \neq 0, C>0\right\} .
$$

Since

and

$$
\left.\frac{d^{2} f}{d \beta^{2}}\right|_{\beta=0^{+}}=2 \eta_{1} \neq 0
$$

$$
\beta \in D_{z_{0}} \Rightarrow-\beta \notin D_{z_{0}}
$$

we have the existence of a unique $\beta=f^{-1}(\eta)=F \eta^{3}, f^{-1}: \Omega \rightarrow D$. Therefore $F=F_{1}(\eta)=\eta^{-3} f^{-1}(\eta)$ is analytic in $\Omega$, and

$$
\frac{d F_{1}}{d \eta}=-3 \eta^{-4} f^{-1}(\eta)+\eta^{-3} \frac{1}{\frac{d f}{d \beta}\left(f^{-1}(\eta)\right)}
$$

tends to infinity as $\eta \rightarrow \eta_{0}$, that is as $\beta \rightarrow 0$. Thus there exists an analytic inverse: $\eta=\eta_{1}(F) ; \eta_{1}: D^{1} \rightarrow \Omega$, where $D^{1} \subset D_{z_{1}}$, for some $z_{1}>0$. Of course from (34) we have the continued resonance in the disk $D^{1}$ :

$$
E(F)=E_{k, k, m}(F)=-\frac{1}{2} \eta_{1}^{2}(F) .
$$

\section{DB Summability and Oppenheimer-Bender-Wu Asymptotics}

The following theorem depends on the analyticity in the perturbation parameter proved in the above sections and on the known sectorial summability $[15,14]$. 
Theorem-7. The eigenvalues $E_{\phi}\left(g^{2}\right)=E\left(g^{2} e^{i \phi}\right), \mathfrak{R} g^{-2}>R^{-1}$, of the quartic anharmonic oscillator (1) are Borel summable

a) in the ordinary sense for $|\phi|<\pi$;

b) in the distributional sense for $\phi=\pi$.

This implies that the Borel transform $B(t)$ is analytic in the cut plane with exponential bound:

$$
\forall \varepsilon>0, \exists C, \gamma:\left|B\left(t e^{i \theta} \pm i \varepsilon\right)\right| \leqq C \exp (\gamma t), \forall \theta: \pm \theta \in[0, \pi] .
$$

Similarly the eigenvalues $E_{k, k, m}\left(F e^{i \phi}\right)$ of the Stark effect operator (32) are Borel summable in the ordinary sense for $0<\phi<\pi$, in the distributional sense for $\phi=0$, and $\phi=\pi$.

Proof. Theorem 1 allows us to apply the criterion for DB summability given in [7]: indeed it essentially requires analyticity in some disk $\mathfrak{R}\left(g^{-2}\right)>R^{-1}$, together with the well-known estimates of the remainders

$$
\left|\sum_{n=0}^{N} c_{n} g^{2 n}-E_{\phi}\left(g^{2}\right)\right| \leqq k a^{n} n !|g|^{2 n}
$$

in any sector (2), where the constants $k, a$ can depend on $\varepsilon_{0}$ (see e.g. [20] for the standard proof of such estimates). Thus DB summability is proved for the quartic oscillator resonances and, in an analogous way, for the Stark resonances.

Since the eigenvalues $E\left(g^{2}\right)$ continued to $\arg \left(g^{2}\right)=\pi$ are non-modal eigenvalues ("resonances") of the formal operator $p^{2}+\mathbf{x}^{2}-|g|^{2} \mathbf{x}^{4}$, the above DB summability result has the consequence of linking in a stricter way the position and the width of such "resonances" with the perturbation series. In particular the following theorem, which connects such different types of asymptotics, depends on DB summability by making use of the direct and inverse Borel transform. For sake of simplicity, we analyze the expansion only up to the second order.

Theorem 8. Let $\Phi(z)=z^{-1} \int_{0}^{\infty} B(t+i 0) \rho(t / z) d t$ be an upper DB sum with respect to the measure $\rho_{\alpha}(t)=e^{-t} t^{\alpha}$ with asymptotic expansion $\sum_{n=0}^{\infty} c_{n} z^{n}$ and with Borel transform locally defined by

$$
B(t)=\sum_{n=0}^{\infty} c_{n}\left(\mu_{n}\right)^{-1} t^{n}, \quad \mu_{n}=\int_{0}^{\infty} t^{n} \rho_{\alpha}(t) d t=\Gamma(n+\alpha+1) .
$$

Let $f(z)=z^{-1} \int_{0}^{\infty} \rho_{\alpha}(t / z) \mathfrak{R} B(t+i 0) d t$, and $g(z)=z^{-1} \int_{0}^{\infty} \rho_{\alpha}(t / z) \mathfrak{F} B(t+i 0) d t$ be the DB sum and the discontinuity respectively.

Let us assume that $B(t)$ has convergence radius 1 , with the only singularities $t= \pm 1$ on the circle $|t|=1$ and let us distinguish two cases:

(a) the singularity is only at $t=1$,

(b) $B(t)=\overline{B(-t)}$.

Then the following facts are equivalent:

i) $\mathfrak{F} B(t+i 0)=\pi \delta(t-1)+b_{1} \pi \theta(t-1)+O((t-1) \theta(t-1))$, as $t \rightarrow 1$,

ii) (a) $c_{n}=\Gamma(n+\alpha+1)\left\{1+b_{1} / n+O\left(1 / n^{2}\right)\right\}$ as $n \rightarrow \infty$,

ii) (b) $c_{2 n}=\Gamma(2 n+\alpha+1)\left\{1+b_{1} / n+O\left(1 / n^{2}\right)\right\}$ as $n \rightarrow \infty, c_{2 n+1}=0$,

iii) $g(z)=(\pi / z) \rho_{\alpha}(1 / z)\left\{1+b_{1} z+O\left(z^{2}\right)\right\}$ as $z \rightarrow 0+$. 
Formulas corresponding to i), ii), iii) can be obtained under dilation $t \rightarrow \sigma t . \sigma>0$ in the Borel transform $B$, which corresponds to a shift of the positive $t$-singularity from 1 to $\sigma^{-1}$.

Proof. Let us consider the case (a) (the case (b) is analogous).

i) implies ii).

Indeed i) is due to a polar and a logarithmic singularity in the Borel transform: i.e. there exists a regular function $C(t),|C(t)| \leqq C, 0 \leqq t \leqq 1$ so that, for $0 \leqq t<1$,

$$
\begin{aligned}
B(t) & =(1-t)^{-1}+b_{1} \log (1-t)+C(t)\{(1-t) \log (1-t)-(1-t)\} \\
& =1+\sum_{n=1}^{\infty}\left(1-b_{1} / n\right) t^{n}-C(t) \int_{0}^{t} \log (1-\tau) d \tau \\
& =1+\sum_{n=1}^{\infty}\left\{\left(1+b_{1} / n\right) t^{n}-C(t) t^{n+1} / n(n+1)\right\} \\
& =1+\left(1+b_{1}\right) t+\sum_{n=2}^{\infty}\left\{1+b_{1} / n+\beta_{n}\right\} t^{n},
\end{aligned}
$$

where

$$
\beta_{n}=O\left(1 / n^{2}\right)
$$

depends on $C(t)$. Hence the asymptotics of $f(z)$, as $z \rightarrow 0+$, follows:

$$
\begin{aligned}
f(z)= & \int_{0}^{\infty} \rho_{\alpha}(t / z) \mathfrak{R} B(t+i 0) d t / z \\
= & \int_{0}^{1 / z} \rho_{\alpha}(\tau)\left\{1+\left(1+b_{1}\right) \tau z+\sum_{n=2}^{N}\left(1+b_{1} / n+\gamma_{n}\right)(\tau z)^{n}\right\} d \tau \\
& +O\left(z^{N+1}\right)+\int_{1 / z}^{\infty} \rho_{\alpha}(t / z) \mathfrak{R} B(t+i 0) d t / z \\
= & \sum_{n=0}^{N} c_{n} z^{n}+O\left(z^{N+1}\right)+O\left(\rho_{\alpha}(1 / z)\right),
\end{aligned}
$$

where $c_{n}$ obeys (ii) because of (42).

ii) implies i).

By (ii), for $0 \leqq t<1$,

$$
B(t)=\sum_{n=0}^{\infty}\left\{c_{n} / \Gamma(n+\alpha+1)\right\} t^{n}=\sum_{n=0}^{\infty}\left\{1+b_{1} / n+\beta_{n}\right\} t^{n},
$$

where $\beta_{n}=O\left(1 / n^{2}\right)$, as $n \rightarrow \infty$. Therefore there is some regular function $C(t)$ such that

$$
B(t)=(1-t)^{-1}+b_{1} \log (1-t)+C(t)\{(1-t) \log (1-t)-(1-t)\},
$$

whence i) follows.

i) implies iii). 
Employing the obvious notation

$$
\begin{aligned}
\mid g(z)- & g_{1}(z) \mid \\
= & \left|z^{-1} \int_{0}^{\infty} \rho_{\alpha}(t / z) \mathfrak{F}\left\{B(t+i 0)-(t+i 0-1)^{-1}-b_{1} \log (1-t-i 0)\right\} d t\right| \\
& \leqq c\left|\int_{1}^{1+\varepsilon} \rho_{\alpha}(t / z)(t-1) d t / z\right|+\left|\int_{1+\varepsilon}^{\infty} \rho_{\alpha}(t / z) \mathfrak{F} B(t+i 0) d t / z\right| \\
\leqq & c e^{-1 / z} / z^{\alpha}\left\{\int_{0}^{\varepsilon} e^{-t / z}(1+t)^{\alpha} t d t / z+\int_{\varepsilon}^{\infty} e^{-t / z}(1+t)^{\alpha}|\mathfrak{F} B(t+i 0+1)| d t / z\right\} \\
\leqq & \rho_{\alpha}(1 / z)\left\{\int_{0}^{\varepsilon} z e^{-t}(1+z t)^{\alpha} t d t\right. \\
& \left.+e^{-\varepsilon / 2} \int_{0}^{\infty} e^{-t / z}(1+\varepsilon+t)^{\alpha}|\mathfrak{F} B(t+i 0+1+\varepsilon)| d t / z\right\} \\
\leqq & \rho_{\alpha}(1 / z)\left(c_{1} z+z^{-1} c_{2} e^{-\varepsilon / z}\right) \leqq c_{3} z^{2} z^{-1} \rho_{\alpha}(1 / z) \\
\leqq & c_{3} z^{-1} \rho_{\alpha-2}(1 / z) .
\end{aligned}
$$

iii) implies i)

First notice that, if $h(t)=H^{\prime}(t)$, an integration by parts shows:

$$
z^{-1} \int_{0}^{\infty} \rho_{\alpha}(t / z) H(t) d t=O(z) z^{-1} \int_{0}^{\infty} \rho_{\alpha}(t / z) h(t) d t .
$$

Now, assume for sake of simplicity $\alpha=0$. A discontinuity having the principal behaviour of iii), can be written as

$$
g(z)=\pi z^{-1} e^{-1 / z} G(z), \text { where } G(z) \text { is regular, near } z=0 .
$$

The inverse Borel transform of $g(z)$ is essentially given [7] by the Riemann-Fourier inversion formula of the Laplace transformation, regarded in the variable $w=z^{-1}$, so that for $t>0$ :

$$
\begin{aligned}
\mathfrak{F} B(t+i 0)= & F(2 \pi i)^{-1} \int_{\Re z^{-1}=R^{-1}} e^{t / z} g(z) z^{-1} d z \\
= & \mathfrak{F}(2 \pi i)^{-1} \int_{r^{-1}-i \infty}^{r^{-1}+i \infty} e^{(t-1) w}\left(C_{0}+C_{1} w^{-1}+\ldots\right) d w \\
= & C_{0} \mathfrak{F}(1-t-i 0)^{-1}+C_{1} \mathfrak{F} \log (1-t-i 0) \\
& +C_{2} \mathfrak{F} \int_{0}^{t+i 0} \log (1-\tau) d \tau+\ldots,
\end{aligned}
$$

where $\sum C_{n} z^{n}$ is the convergent expansion of $G(z)$ at $z=0$, and the integral is performed on the path $\mathfrak{R} z^{-1}=r^{-1}$, with $r$ less than the radius of convergence of $G(z)$.

Hence the principal behaviour in i) is proved, as well as the other singular behaviours as $t \rightarrow 1$ by the above remark (47). 


\section{References}

1. Andrianov, A.: Ann. Phys. 140, 82 (1982)

2. Bender, C., Wu, T.T.: Phys. Rev. Lett. 21, 406 (1968)

3. Bender, C., Wu, T.T.: Phys. Rev. Lett. 16, 461 (1971)

4. Bender, C., Wu, T.T.: Phys. Rev. D 7, 1620 (1973)

5. Breen, S.: Rutgers University Thesis (1980), unpublished

6. Buslaev, V., Grecchi, V.: Equivalence of unstable anharmonic oscillators and double wells. Bologna preprint (1992)

7. Caliceti, E., Grecchi, V., Maioli, M.: Commun. Math. Phys. 104, 163-174 (1986)

8. Caliceti, E., Grecchi, V., Maioli, M.: Commun. Math. Phys 113, 625-648 (1988)

9. Caliceti, E., Grecchi, V., Maioli, M.: Commun. Math. Phys. 113, 173-176 (1987)

10. Caliceti, E., Grecchi, V., Maioli, M.: Atti Sem. Mat. e Fis. Univ. di Modena XXXVI, 85-93 (1988)

11. Caliceti, E., Maioli, M.: Ann. Inst. H. Poincaré Sect. A, 38, 175-186 (1983)

12. Candelpergher, B.: Une introduction à la résurgence. Gazzette des Mathématiciens, Société Mathématique de France 42, 136 (1989)

13. Graffi, S., Grecchi, V.: Phys. Lett. B 121, 410 (1983)

14. Graffi, S., Grecchi, V.: Commun. Math. Phys. 62, 83 (1978)

15. Graffi, S., Grecchi, V., Simon, B.: Phys. Lett. B32, 631 (1970)

16. Harrell, E., Simon, B.: Duke Math. J. 47, 845 (1980)

17. Herbst, I.: Commun. Math. Phys. 64, 179 (1978)

18. Hunziker, W., Vock, E.: Commun. Math. Phys. 83, 281 (1982)

19. Hunziker, W.: Helv. Phys. Acta 61, 257-304 (1988)

20. Reed, M., Simon, B.: Methods of modern mathematical physics. New York: Academic Press 1978

21. Simon, B.: Int. J. Quantum Chemistry 21, 3-25 (1982)

22. Simon, B.: Bull. Am. Math. Soc. 24, 303 (1991)

23. 't Hooft, G.: The ways of subnuclear physics. Proceedings of the international school of subnuclear physics. Erice (1979), Zichichi A. (ed.), pp. 943-971. New York: Plenum 1979

Communicated by B. Simon 
